\title{
THE FEASIBILITY OF A COGNITIVE BEHAVIOURAL THERAPY GROUP FOR MEN WITH MILD/MODERATE COGNITIVE IMPAIRMENT
}

\author{
Tara Kipling and Michelle Bailey \\ North East Essex Mental Health NHS Trust, Colchester, U.K. \\ Georgina Charlesworth \\ NEEMHT, and University of East Anglia, Norwich, U.K.
}

\begin{abstract}
Memory aid groups have often been used as a method for teaching mnemonic strategies to older adults in early stages of dementia. This study describes the use of CBT to address unhelpful memory-related beliefs in three older men with mild/moderate dementia and associated low mood or anxiety. The members were able to participate and engage in the sessions, and changes in behaviour, cognition and affect were monitored over the course of a 7 week group intervention. Recommendations are made for further research.
\end{abstract}

Keywords: Memory, group, CBT, dementia, elderly.

\section{Introduction}

Complaints of poor memory are common in old age. Interventions such as memory aid groups have typically focused upon practical management strategies and techniques for mnemonic enhancement. Such memory training can be beneficial, particularly in group format, but there are difficulties with attrition, maintenance of gains and an over-expectation of outcome (Scogin, 1992). Furthermore, groups can "feel like loading a shotgun with lots of pellets and hoping some hit the target" (Allen, 1996). It seems likely that the most beneficial aspects are not the mnemonic or behavioural management strategies but the non-specific group factors that impact on group members' affective response to their memory problems. Cognitive psychology literature would suggest that addressing affect is not only desirable but essential, as negative affective states interfere, directly or indirectly, with memory functioning (Deptula, Singh, \& Pomara, 1993).

In this study Padesky and Mooney's (1990) generic cognitive therapy model was used as the basis for a structured group programme, addressing maladaptive memory-related

Reprint requests and requests for extended report to Tara Kipling, Doctoral Programme in Clinical Psychology, School of Health Policy and Practice, University of East Anglia, Norwich NR4 7TJ, U.K. 
beliefs. It was hypothesized that the group would: reduce negative affect; increase knowledge regarding memory functioning; and, stimulate adaptive behavioural changes.

\section{Method}

\section{Subjects}

Participants were selected during a multi-disciplinary team meeting at a Mental Health Trust Day Hospital. Selection criteria were: subjective complaint and objective evidence of memory difficulties, living with a full time carer. Three men were chosen. Each had a diagnosis of probable dementia and were aged 75 to 84 years $($ mean $=80.3)$. Baseline Mini Mental State Examination (MMSE; Folstein, Folstein, \& McHugh, 1975) scores ranged between 19 to 23 (mean =21). None were taking cognitive enhancing medication. All three members attended every session.

\section{Measures}

Self-report 7-point semantic differential scales (Emmerson \& Bucks, 1997) were used pre- and post-group. There are seven dimensions, under three headings, namely: mental state (confidence, mood, anxiety); cognitive functioning (memory, concentration); and behaviour (ability to manage own affairs, independence). In addition to these quantitative measures, spontaneous reports of behavioural changes were acknowledged and recorded by group facilitators.

\section{Intervention}

Seven weekly sessions of one hour duration were facilitated by an Assistant Psychologist (TK) and Occupational Therapist (MB). The aim was to provide a safe environment where people felt relaxed enough to share their thoughts and feelings about the memory difficulties and learn how these could be exacerbated by the reactions of oneself and others, including family.

Each session contained a psycho-educational component followed by group participation, practical task and finally a summary. Handouts were provided as a reminder and to share with their partners. The first session focused on the multifaceted nature of memory. Subsequent sessions focused on aspects of the Padesky and Mooney's model (1990) and included: memory and mood; memory and body sensations; memory and thoughts; memory and situations, behaviours and actions; and the final summary session presented the complete model. Group exercises included: relaxation, identifying negative automatic thoughts about memory in particular, generating alternatives, problem solving.

Sessions were designed to counter unhelpful beliefs about memory, such as "In order to engage in social activities we must remember names and faces" (a belief implicitly reinforced by memory aid groups), or "I'm the only one who forgets X". The latter belief can, in the first author's experience, dramatically impact upon self-esteem and 
Table 1. Age, MMSE scores and change on semantic differential scales pre- and post-group for each of the three subjects

\begin{tabular}{lccc}
\hline & Subject 1 & Subject 2 & Subject 3 \\
\hline Age & 75 & 84 & 81 \\
MMSE 1 & 20 & 23 & 19 \\
MMSE 2 & 16 & 25 & 21 \\
Mental state & & & \\
$\quad$ Confidence & +1 & 0 & -1 \\
Mood & +1 & 0 & +1 \\
$\quad$ Anxiety & -1 & -3 & -1 \\
Cognitive functioning & & & \\
$\quad$ Concentration & 0 & +1 & 0 \\
$\quad$ Memory & 0 & 0 & 0 \\
Behaviour & & & \\
$\quad$ Managing affairs & -1 & -1 & -1 \\
$\quad$ Independence & 0 & -1 & -2 \\
\hline
\end{tabular}

general functioning and unless it or underlying beliefs are addressed it can be a barrier to coping strategy enhancement.

\section{Results and discussion}

Overall, the members were able to participate and engage in the sessions.

\section{Mental state}

All three participants reported an increased ability to relax. This is labelled in Table 1 as the anxiety continuum. Also, there was a positive change for two participants on the mood scale.

\section{Cognitive functioning}

Subject 2 alone rated his concentration more highly, and there were no other changes reported in memory or concentration on the semantic differential scales. Scores on the MMSE improved by 2 points for subjects 2 and 3, but decreased by 4 points for subject 1. All three participants gave spontaneous examples of using their memory more rather than relying on others to remember for them.

\section{Behaviour changes}

All three resigned themselves to the fact that they could no longer manage their own affairs as indicated by the negative change scores. This result, coupled with the improved mood and anxiety scores, suggests that reduced abilities can be acknowledged 
without reducing self-esteem. In addition to the semantic differential scores, participants were asked about behavioural changes. Reports included: going shopping, greeting people even when they could not recall the person's name, attending parties, and answering the telephone.

\section{Follow-up}

Subject 1 experienced rapid cognitive and physical decline. He moved into residential care and died within 6 months of the group. A follow-up group session was held three months after session 7 and attended by subjects 2 and 3. They remembered the group, one facilitator's name and that they were not alone in experiencing difficulty with their memory. Self-report ratings were not repeated. However, group members reported maintenance of behavioural changes. Subject 3 commented on how he found talking to people who also had memory problems helpful, and how he continued to employ relaxation techniques. Subject 2 had left the Day Hospital and was attending a social club.

In summary, the results show that a group approach to memory difficulties using a cognitive behavioural model is feasible and may be beneficial. By viewing each pairing of elements in Padesky's generic model, group members were able to build an understanding of the complete model. Self-report on semantic differential scales demonstrated an improvement in mood. Participants described changes in behaviour, and self-beliefs, and demonstrated a greater understanding of how mood can affect memory functioning.

The study is limited by small numbers, and a further evaluation with several therapy groups is required. Morris (1994) notes that effective group work with older adults with dementia should involve relatively small groups. This is to counteract the known problems with working memory and divided attention in these patients. A more detailed evaluation of cognitive, behavioural, emotional and physiological changes would be necessary for future studies using controlled research design.

\section{Acknowledgements}

The authors would like to thank Anne Fawcett and the reviewers for comments on an earlier draft of this paper.

\section{References}

Allen, C. (1996). The effectiveness of memory aid groups. PSIGE Newsletter, 56, 15-19.

Deptula, D., Singh, R., \& Pomara, N. (1993). Aging emotional states and memory. American Journal Psychiatry, 150, 429-434.

Emerson, C., \& Bucks, R. (1997). Insight in dementia: A pilot study to examine three measurement methods. PSIGE Newletter, 61, 10-15.

Folstein, M. F., Folstein, S. E., \& McHugh, P. R. (1975). "Mini-Mental State": A practical method for grading the cognitive states of patients for the clinician. Journal of Psychiatric Research, 12, 189-198.

Morris, R. G. (1994). Working memory in Alzheimer-type dementia. Neuropsychology, 8, 544554. 
Padesky, C. A., \& Mooney, K. A. (1990). Clinical tip presenting the cognitive model to clients. International Cognitive Therapy Newsletter, 6, 11-12.

Scogin, F. (1992). Memory training for older adults. In G. M. M. Jones \& B. M. C. Miesen (Eds.), Care giving in dementia. London: Routledge. 